ISSN 1676-3742

\title{
Los manuscritos de Qumrán: problemas de taxonomía 1
}

\author{
The Dead Sea Scrolls: \\ taxonomical problems
}

Florentino García Martínez

\section{Resumen}

El artículo intenta presentar un panorama general del contenido de los textos de Qumrán a partir de los problemas que la clasificación de los distintos manuscritos nos presentan. La clasificación de las ediciones oficiales atestiguan el pan-biblismo y pan-esenismo corrientes en los años cincuenta. Pero cuando todos los textos han sido pubicados, esas clasificaciones crean problemas. Una comparación de la organización de los materiales en mi traducción de los manuscritos de Qumrán, Textos de Qumrán, publicada en castellano en 1992, con la de la edición bilingüe (hebreo-arameo e inglés) de la Study Edition, publicada en 2000, permitirá comprender que poner un nombre a una obra literaria o cambiárselo no es una actividad anodina e inocua sino que puede condicionar profundamente la manera de comprenderla.

Palabras claves: Manuscritos de Qumrán. Textos bíblicos y no bíblicos. Textos sectarios y no sectarios. Problemas de clasificación.

\footnotetext{
${ }^{1}$ Texto de una conferencia dada en la Fundación Juan March de Madrid el 19 de mayo de 2015. He mantenido el estilo oral de la presentación, limitándome a añadir las referencias indispensables.
} 


\section{Summary}

The article gives a general description of the contents of the Dead Sea Scrolls by discussing the classification problems they have created lately. The original classification show the pan-biblical and pan-essene understanding current in the fifties. But when all the texts have been published, these classification creates serious problems. A comparison of the organization of the material in Textos de Qumran, published in 1992, with the bilingual The Dead Sea Scrolls Study Edition of 2000 should allow to understand that giving a name or changing the name given to a composition can deeply influence the understanding of the work in question.

Keywords: Dead Sea Scrolls. Biblical and non-biblical texts. Sectarian and non-sectarian texts. Classification problems.

\section{Introducción}

Una buena forma de describir el contenido de los manuscritos de Qumrán sin entrar en demaisados detalles tecnicos puede ser la de presentar las polémicas más recientes entre los especialistas de la materia que conciernen los problemas de clasificación de los distintos manuscritos, problemas que han salido a la luz una vez que todos los textos han sido publicados. ${ }^{2}$ Un recorrido por las diversas maneras de etiquetar los contenidos de los distintos manuscritos en las ediciones oficiales nos permitirá adquirir un conocimiento de esos contenidos más conforme con la realidad histórica de la colección.

Los títulos que describían el contenido de las obras contenidas en los manuscritos para aquellos que las coleccionaron y preservaron que han llegado hasta nosotros son muy pocos. En cuatro casos el título se ha conservado al dorso del manuscrito: 1QS Regla de la Comunidad y de..., ${ }^{3} 4 \mathrm{Q} 8 \mathrm{c}$ Bereshit

\footnotetext{
${ }^{2}$ El estudio más reciente y más lúcido es el de NAJMAN, H y TIGCHELAAR, E. “A Preparatory Study of Nomenclature and Text Designation in the Dead Sea Scrolls". Revue de Qumrân 103 (2014), pp. 305-325.

${ }^{3}$ Editado por MILIK, J.T. En Qumrân Cave 1. DJD1; Oxford: Clarendon, 1955, pp. 107-108, pl. XXII
} 
(la primera palabra hebrea de Génesis), ${ }^{4}$ 4Q249 Midrás del libro de Moisés, ${ }^{5}$ y 4Q504 Palabras de los Luceros. ${ }^{6}$ En una decena de casos el título original se puede deducir de las primeras palabras de la obra (el incipit), como es corriente en la literatura hebráica. Estos títulos pueden referirse: - al contenido general de la composición, como en 4Q298: Palabras del Sabio a los hijos de la aurora, ${ }^{7}$ o en 4Q529: Palabras del escrito que dijo Miguel, ${ }^{8}$ o en 4Q543: Copia del escrito de las palabras de la visión de Amran; ${ }^{9}$ - o pueden designar lo que hoy definiríamos como el género literario de la obra: Serek (Regla) en 1QS Regla de la Comunidad ${ }^{10}$ y 1QSa Regla de la Congregación; ${ }^{11}$ Pesher en 4Q180: Pesher sobre los períodos, ${ }^{12}$ o Midrash en 4Q258 (el comienzo de una de las copias de la Regla de la Comunidad de la cueva 4: Interpretación para el Instructor; ${ }^{13}$ - o pueden referir el contenido de la composición a determinados personajes del pasado de Israel: Moisés o Amram, por ejemplo. En algunas ocasiones los manuscritos nos han conservado referencias a los títulos de determinadas obras, pero estas obras no se han conservado, como es el caso del Libro de Noé, mencionado en 1QGénesis Apócrifo 5: 29, o el famoso Libro de la meditación (Sefer Hagu) en el que los jóvenes deben ser educados según la Regla de la Congregación 1: 5 y del que se nos dice en el

\footnotetext{
${ }^{4}$ Editado por DAVILA, J.R. En Qumran Cave 4. VII: Genesis to Numbers. DJD 12; Oxford: Clarendon, 1995, pp. 63-64, pl. XII

${ }^{5}$ Editdo por PFANN, S. En Qumran Cave 4. XXV: Halakhic Texts. DJD 35; Oxford: Clarendon, 1999, pp. 1-24, pl.I- II.

${ }^{6}$ Editado por BAILLET, M. En Qumrân grotte 4.III. DJD 7; Oxford: Clarendon, 1982, pp.137168, pl. XLIX-LIII.

${ }^{7}$ Editado por PHANN, S.J. y KISTER, M. En Qumran Cave 4. XV: Sapiential Texts, Par I. DJD 20; Oxford: Clarendon, 1997, pp. 1-30, pl. I-II.

${ }^{8}$ Editado por PUECH, E. En Qumrân grotte 4. XXII:Textes araméens première partie. DJD 31; Oxford: Clarendon, 2001, pp. 1-8, pl. I.

${ }^{9}$ Editado por PUECH, E.En DJD 31(citado en la nota 8); p. 292, pl. XVI

${ }^{10}$ Editado por BURROWS, M. En The Dead Sea Scrolls of St. Mark's Monastery: Volume II. Fascicle 2. New Haven: The American Schools of Oriental Research, 1951.

${ }^{11}$ Editado por BARTHÉLEMY, D. En DJD 1 (citado en la nota 3); pp. 107-118, pls XXIII-XXIV.

${ }^{12}$ Editado por ALLEGRO, J.M. En Qumran Cave 4. I. DJD 5; Oxford: Clarendon, 1969, pp. 77-79, pl. XXVIII.

${ }^{13}$ Editado por ALEXANDER, P. y VERMES, G. En Qumran Cave 4. XIX: Serek ha-Yahad and Two Related Texts. DJD 26; Oxford: Clarendon, 1998, 93 pl. X.
} 
Documento de Damasco ${ }^{14}$ 13:2-3 "Y en un lugar de diez, que no falte un sacerdote instruído en el libro de HAGU; y por su autoridad todos serán gobernados."

Puesto que los títulos originales de la inmensa mayoría de las composiciones recuperadas era desconocido los investigadores de la primera generación, a la hora de clasificar y dar títulos a las obras encontradas, se vieron obligados a partir de lo que a ellos les era conocido. Y lo que ellos sabían dependía por una parte de una visión canónica de la literatura religiosa (que dividía los textos entre lo que es bíblico y lo que no lo es), y por otra parte de una concepción determinda de la comunidad que había coleccionado la biblioteca que era identificada sin más con los esenios. Por lo tanto los primeros editores en los años 50 encuadraron todos los manuscritos descubiertos en unas categorías que eran pan-bíblicas y pan-esenias.

\section{Contexto hermenéutico de las de las primeras publicaciones}

En la publicación del primer volúmen de la serie oficial de la edición de los textos, Discoveries in the Judaean Desert (serie abreviada en las referencias bibliográficas por las siglas DJD), en el que fueron publicados los materiales procedentes de la Cueva 1 propiedad del Museo Rockefeller, sobre una propuesta de Milik, discutida y aprobada por las American Schools of Oriental Research, se nos da una primera gran división del material hallado: obras canónicas (los textos bíblicos) y obras no-canónicas (los textos que no forman parte de la Biblia); estos libros no bíblicos son a su vez divididos en tres categorías: comentarios (designados por la sigla $\mathrm{p}=$ pesher), apócrifos, y obras de la "biblioteca esenia." En la categoría de "comentarios" se dan como ejemplos 1QpHab, 1QpPs 68 (1Q16) y 4QpSa 37. En la categoría "apócrifos" se citan 1QLamech (Apocalípsis de Lamec, que será finalmente publicado com Génesis apócrifo), 'QJubileos ('Q'V y 'Q', ('^QTestamento de Leví ('Q ' ' '), 4QTestamento de Leví, 1QPalabras de Moisés (1Q22) (una composición semejante a Jubileos, pero inspirada en el Deuteronomio y no en Génesis) y 1QLibro de los misterios (1Q27) (una composición sapiencial de la que hay otras dos o tres copias en la

\footnotetext{
${ }^{14}$ Los dos ejemplares de la Genizah de El Cairo fueron editados por SCHECHTER, S. En Documents of Jewish Sectaries. Vol. 1: Fragments of a Zadokite Work edited from Hebrew manuscripts in the Cairo Genizah now in possession of University Library, Cambridge and provided with an English translation, introduction and notes. Cambridge : Combridge University Press, 1910. Los ocho ejemplares de la Cueva 4 fueron editados por BAUMGARTEN, J.M. En Qumran Cave 4. XIII: The Damascus Document. DJD 18; Oxford: Clarendon, 1996.
} 
cueva 4). En la categoría de textos de la biblioteca esenia, se mencionan 1QRegla de la Comunidad, 1QRegla de la Congregación, 1QColección de Bendiciones, 1QRegla de la Guerra, 1QHimnos y el Documento de Damasco, y a estas composiciones esenias se les atribuyen títulos hebreos como Serek, Milhama y Hodayoth. ${ }^{15}$ Aunque entre los ejemplos citados hay algunos provenientes de la cueva 4, queda claro que el contexto hermenéutico, el marco de interpretación general, se había formado tomando como base los manuscritos entonces conocidos, es decir los siete grandes manuscritos procedentes de la Cueva 1, tanto los publicados por la Universidad Hebrea, como los publicados por las Americal Schools of Oriental Research (los manuscritos mejor conservados) y los textos incluídos en el volúmen primero de la serie oficial DJD (más fragmentarios pero semejantes a los otros en su contenido).

Es decir, que el contexto hermenéutico con el que se clasificaron los textos había sido formado en base a un número limitado de textos que presentaban un perfil particular. Estos textos eran: - dos copias del libro de Isaías, ${ }^{16}$ con numerosas variantes, desde luego, pero fundamentalmente del mismo tipo textual que el texto masorético; - una composición aramea, el Génesis Apócrifo, ${ }^{17}$ que en parte ampliaba la historia de los Patriarcas con nuevos detalles y en parte traducía casi literalmente el texto masorético, una composición semejante a otros apócrifos judíos ya conocidos, como el Libro de los Jubileos o las Antigüedades Judías del Pseudo Filón; - y, junto a estos dos manuscritos bíblicos y uno para-bíblico, cuatro composiciones totalmente desconocidas (la Regla de la Comunidad y el Pesher de Habacuc, ${ }^{18}$ así como la Regla de la Guerra y los Himnos o Hodayot ${ }^{19}$ ) que nos revelaban por vez

${ }^{15}$ DJD 1 (citado en la nota 3): pp. 42-43.

${ }^{16}$ Uno (1QIsa ${ }^{a}$ ) editado por BURROWS, M. En The Dead Sea Scrolls of St. Mark's Monastery: Volume I. New Haven: The American Scools of Oriental Research, 1950, y el otro (1QIsa ${ }^{\mathrm{b}}$ ) editado por SUKENIK, E.L. En The Dead Sea Scrolls of the Hebrew University. Jerusalem: Magnes Press/The Hebrew University, 1955. Ambos han sido recientemente reeditados por ULRICH, E. y FLINT, P.W. En Qumran Cave 1.II: The Isaiah Scrolls. DJD 32; Oxford: Calrendon 2010.

${ }^{17}$ Editado por AVIGAD, N. y YADIN, Y. En A Genesis Apocryphon. A Scroll from the Wilderness of Judaea. Jerusalem: Magnes Press/Heikal ha-Sefer, 1956. Una edición más recientemente y más complete es la de MACHIELA, D. The Dead Sea Genesis Apocryphon. STDJ 79; Leiden: Brill, 2009.

${ }^{18}$ Editados por BURROWS, M. (ver las notas 10 y 16)

${ }^{19}$ Editados por SUKENIK,E.L. (citado en la nota 16). La edición más reciente y más completa de los Hodayot es la de STEGEMANN, H. y SCHULLER, E. En 1QHodayot ${ }^{a}$ with incorporation of 1QHodayot ${ }^{b}$ and 4QHodayot ${ }^{a-f}$. DJD 40; Oxford: Clarendon 2009. 
primera la existencia, el pensamiento religioso, la manera de interpretar el texto bíblico y la forma de vida de un grupo judío del que previamente no sabíamos nada.

La Regla de la Comunidad nos permitía descubrir la existencia de un grupo particular, con sus normas propias, su estructura jerarquizada y con elementos doctrinales característicos y exclusivos, como el dualismo, la predestinación, el mesianismo bicéfalo, etc. Un grupo que no correspondía a nada de lo que sabíamos de los grupos judíos de la época. Los Himnos nos ofrecían una poesía fuertemente personal y de grande densidad teológica, perfectamente compatible con las ideas peculiares de la Regla de la Comunidad. El Pesher de Habacuc nos confrontaba con una interpretación del texto bíblico en función de la historia propia y de los conflictos exteriores e interiores del grupo. Y la Regla de la Guerra, nos revelaba la ardiente espera escatológica del grupo, orientado hacia un combate final y definitivo de las fuerzas del mal (los 'Hijos de las Tinieblas') y de las fuerzas del bien (los 'Hijos de la Luz', con los que los miembros del grupo se identificaban). ${ }^{20}$

\section{Publicaciones subsiguientes}

En resumen, pues, estos manuscritos de la Cueva 1, los primeros en ser publicados, formaron el marco hermenéutico en el que irían integrándose las publicaciones sucesivas de otros textos. Esta forma de clasificar los primeros manuscritos descubiertos era completamente normal, puesto que la investigación parte siempre de aquello que es conocido. Más problemático es el hecho de que este contexto hermenéutico permaneció substancialmente invariado y condicionó la manera de designar los textos procedentes de la otras cuevas que poco a poco iban publicándose: DJD 3 con los manuscritos de la cuevas menores aparecido en $1962,{ }^{21}$ DJD 5, el primero dedicado a la cueva 4 que contiene la edición de 4Q158-4Q186, aparecido en 1969, ${ }^{22}$ y DJD 7 con la edición de 4Q483-4Q520, aparecido en 1982. ${ }^{23}$ Estos volúmenes nos

${ }^{20}$ Para una descripción detallada de estos manuscritos se puede ver GARCÍA MARTÍNEZ, F. "Textos de Qumrán”. En ARANDA PÉREZ, G., GARCÍA MARTÍNEZ, F. y PÉREZ FERNÁNDEZ, M. Introducción al Estudio de la Biblia, Vol. IX: Literatura Judía Intertestamentaria. Estella: Verbo Divino, 1996, pp. 17-241.

${ }^{21}$ BAILLET, M., MILIK,J.T. y DE VAUX, R., Les 'Petites Grottes'de Qumrân. Exploration de la falaise. Les grottes 2Q, 3Q, 5Q, 6Q, 7Q à 10Q. DJD 3; Oxford: Clarendon, 1962.

${ }^{22}$ Ver nota 12.

${ }^{23}$ Ver nota 6. 
revelan claramente que junto al pan-biblismo y pan-esenismo otro elemento fundamental empleado en la clasificación y en la nomenclatura de los textos fue su relación con géneros literarios conocidos en la literatura bíblica (literatura sapiencial, legal, apocalíptica, hímnica, etc.) o en la posterior literatura cristiana (Testamento, florilegium, catena, testimonium, etc.) Así, en DJD 3 entre los textos no-bíblicos encontramos junto a textos narrativos, textos proféticos, jurídicos, litúrgicos e hímnicos. En DJD 7, en el apartado "Apócrifos y diversos" encontramos junto a Jubileos, un Testamento de Juda, textos sapienciales, apocalípticos y litúrgicos. Y en DJD 5, que contiene la publicación de todos los pesharim de la cueva 4, encontramos igualmente la edición de 4Q159 Ordenanzas, 4Q174 Florilegio, 4Q175 Testimonia y dos Cadenas exegéticas 4Q177 y 4Q182, títulos claramente derivados de la posterior literatura cristiana.

Cuando, a partir de los años 90, la Israel Antiquities Authority reorganizó el comité internacional de edición y facilitó el acceso de los investigadores a la totalidad de los manuscritos, el contexto hermenéutico permanció substancialmente invariado, como aparece claramente en la explicación que el editor en jefe, Emanuel Tov, ofrece en las secciones "Identifications and Nomenclature of the Texts" y "Literary genres" en el volúmen de Indices de la serie DJD 39. ${ }^{24}$ La distinción básica entre textos bíblicos (los que están en el canon de la Biblia hebrea) y los no bíblicos (todos los demás) permanece intacta, pero en la categoría de textos no-bíblicos se agrupan los textos no por su condición de no sectarios o sectarios (apócrifos o esenios en la nomenclatura de DJD 1), sino por categorías o géneros literarios. ${ }^{25}$ Así DJD $11^{26}$ y DJD

\footnotetext{
${ }^{24} \mathrm{TOV}, \mathrm{E}$. The Texts from the Judaean Desert. Indices and an Introductions to the Discoveries in the Judaean Desert Series. DJD 39; Oxford: Clarendon 2002, pp. 10-14.

25 "The texts from cave 4 are likewise presented according to literary genre, and since the texts from this cave are so numerous, each volume of the non-biblical texts presents a single literary genre from vol. X onwards. In this arrangement, volumes were co-authored by several scholars, such as vols. XIII, XIX, and XXII containing so-called 'parabiblical texts'. Other volumes group sapiential texts (XX, XXXIV), poetical and liturgical texts (XI, XXIX), halakhic texts (XXXV), etc.” DJD 39, 14.

${ }^{26}$ ESHEL, E. y otros, Qumran Cave 4.VI: Poetical and Liturgical Texts. Part I. DJD 11; Oxford: Clarendon 1998.
} 
$29^{27}$ publican manuscritos poéticos o litúrgicos, DJD $20^{28}$ y DJD $34{ }^{29}$ publican textos sapienciales, DJD $35^{30}$ recoge los textos haláquicos otros que las copias del Documento de Damasco publicadas en DJD $18^{31}$. Con DJD 13, ${ }^{32}$ aparece el primer volúmen de textos de una nueva categoría considerada como género literario: los textos "para-bíblicos," que será seguido por DJD $19,{ }^{33}$ por DJD $22^{34}$ y por un cuarto volúmen DJD $30^{35}$ aunque en el título de este volúmen, que contiene la edición del "Pseudo Ezequiel" y del "Apócrifo de Jeremías" los textos "para-bíblicos" son definidos como "pseudo-proféticos". Un vistazo al contenido de los otros tres volúmenes de textos para-bíblicos explica la utilización de esta terminología altamente problemática y que continúa el pan-biblismo y la visión canónica de los años $50 .{ }^{36}$ DJD 13 contiene la edición de todas las copias de Julibeos de la cueva 4 (4Q216-228), los manuscritos 4Q364-367 conocidos como paráfrasis del Pentateuco, publicadas en DJD como "Reworked Pentateuch" y actualmetne considerados como versiones diferentes del Pentateuco, la "Plegaria de Enosh" 4Q369, una paráfrasis de Reyes ¿Qr⿲r y una paráfrasis de Génesis y Exodo 4Q422. DJD 19 contiene los cuatro manuscritos arameos y un manuscrito hebreo de Tobías (4Q196200), dos copias de un "Apócrifo de Moisés" (4Q375-376), un “Apócrifo

${ }^{27}$ CHAZON, E. y otros, Qumran Cave 4.XX: Poetical and Liturgical Texts, Part 2. DJD 29; Oxford: Clarendon, 1999.

${ }^{28}$ ELGVIN, T. y otros, Qumran Cave 4.XV: Sapiential Texts, Part 1. DJD 20; Oxford: Clarendon, 1997.

${ }^{29}$ STRUGNELL, J. y otros, Qumran Cave 4.XXIV: 4QInstruction (Musar leMevin). DJD 34; Oxford: Clarendon, 1999.

${ }^{30}$ BAUMGARTEN, J. y otros, Qumran Cave 4.XXV: Halakhic Texts. DJD 35; Oxford: Clarendon, 1999.

${ }^{31}$ Ver nota 14.

${ }^{32}$ ATTRIDGE, H. y otros, Qumran Cave 4.VIII: Parabiblical Texts, Part 1. DJD 13; Oxford: Clarendon, 1994.

${ }^{33}$ BROSHI, M. y otros, Qumran Cave 4.XIV: Parabiblical Texts, Part 2. DJD 19; Oxford: Clarendon, 1995.

${ }^{34}$ BROOKE, G.J. y otros, Qumran Cave 4.XVII: Parabiblical Texts, Part 3. DJD 22; Oxford: Clarendon, 1996.

${ }^{35}$ DIMANT, D. Qumran Cave 4.XXI: Parabiblical Texts, Part 4: Pseudo-Prophetic Texts. DJD 30; Oxford : Clarendon, 2001.

${ }^{36}$ Sobre los problemas de esta terminología, ver GARCÍA MARTÍNEZ, F. y VERVENNE, M. "Ancient Interpretation of Scripture in Light of the Dead Sea Scrolls." En PIQUER OTERO, A. y TORIJANO MORALES, P. (eds.), Textual Criticism and Dead Sea Scrolls Studies in Honour of Julio Trebolle Barrera: Florilegium Complutensis. JSJS 157: Leiden: Brill, 2012, pp. 83-97 y la literatura allí citada en las notas 18 y 19. 
de Jeremías" (4Q384), un "Pseudo Ezekiel" (4Q391) y toda una serie de textos menores. En DJD 22 encontramos seis manuscritos del "Documento arameo de Leví" (4Q213-214), tres copias de un "Pseudo Daniel arameo" (4Q243-245), un "Apócrifo de Daniel arameo" (4Q246), dos "Apócrifos de Josué" (4Q378-379), un "Apócrifo de Eliseo" (4Q181), cuatro "Comentarios a Génesis" (4Q252-254), así como una serie de textos menores. Este rápido recorrido muestra que la nueva categoría de textos "para-bíblicos" en realidad continúa el mismo pan-biblismo de los primeros años y su visión canónica de los manuscritos. Las designaciones de "para-", "pseudo-," "apócrifo-" y similares indican una valoración peyorativa de los escritos así designados y su evaluación como inferiores y secundarios con relación a los textos bíblicos.

Observando las fechas de publicación de los volúmenes de la serie oficial DJD se tiene una idea clara de la persistencia del cuadro hermenéutico diseñado a mediados del siglo 20 y que ha pervivido hasta bien entrado el siglo 21 .

En la introducción a mi libro Textos de Qumrán, ${ }^{37}$ yo me descartaba del pan-esenismo dominante desde los años 50 presentando la hipótesis sobre los orígenes del grupo qumránico que es conocida como "Hipótesis de Groningen." ${ }^{" 38}$ Pero en lo que concierne al pan-biblismo yo seguía deudor a las ideas recibidas, dividiendo los manuscritos en dos grandes categorías: manuscritos bíblicos, no incluídos ni traducidos en mi libro, y manuscritos no bíblicos, de los que ofrecía la traducción. Y esto a pesar de que desde el congreso de El Escorial de 1991, donde habían sido presentados por primera vez los manuscritos 4Q364-367 como paráfrasis del Pentateuco, yo había comenzado a tener mis dudas. Estas dudas las había articulado en una conferencia, publicada el mismo año, ${ }^{39}$ y también incluída posteriormente en

${ }^{37}$ GARCÍA MARTÍNEZ, F. Textos de Qumrán. Estructuras y Procesos / Serie Religión; Madrid: Editorial Trotta, 1992. Ver en la traducción brasileña, Textos de Qumran. Edição fiel e completa dos Documentos do Mar Morto. Petrópolis: Vozes, 1995, las páginas 37-40.

${ }^{38}$ Presentada originalmente en una comunicación al segundo simposio bíblico español: "Orígenes del movimiento esenio y orígenes qumránicos: Pistas para una solución". publicada en COLLADO BERTOMEU,V. y VILLAR HUESO, V. (ed.). II Simposio Bíblico Español (Córdoba, 1985). Valencia-Córdoba: Fundación Bíblica Española, 1987, pp. 527-556, dada a conocer internacionalmente en un congreso de la academia polaca de ciencias con el título "Qumran Origins and Early History: A Groningen Hypothesis". Folia Orientalia 25 (1988), pp. 113-36, e incluída en el libro publicado con TREBOLLE BARRERA, J. Los Hombres de Qumrán: Literatura, estructura social y concepciones religiosas. Estructuras y Procesos / Serie Religión; Madrid: Editorial Trotta, 1993. En la traducción brasileña, Os homens de Qumran. Literatura, estrutura e concepções religiosas. Petrópolis: Vozes, 1996, pp. 94-123.

${ }^{39}$ GARCÍA MARTÍNEZ, F. “Las fronteras de «lo bíblico»”. Scripta Theologica 23 (1991), pp. 759-784. 
el libro Los hombres de Qumrán. ${ }^{40}$ En ese artículo señalaba que los nuevos manuscritos diluían las fronteras entre lo bíblico y lo no-bíblico puesto que nos ofrecían textos de los que no podíamos decir si estaban en camino de llegar a ser bíblicos, si eran formas distintas de textos bíblicos o si ya no eran textos bíblicos.

A pesar de estas dudas, la agrupación de los manuscritos en diferentes categorías que yo hice en la edición y traducción publicada en 1992, era claramente deudora a las ideas entonces corrientes y agrupaba los textos en función de su contenido o del supuesto género literario. Un recorrido por los títulos de los distintos capítulos de Textos de Qumrán lo muestra claramente: Reglas, Textos haláquicos, Literatura de contenido escatológico, Literatura exegética, Literatura para-bíblica, Textos poéticos, Textos litúrgicos, Textos astronómicos, calendarios y horóscopos, y un capítulo final dedicado al Rollo de cobre. En el prólogo yo explicaba esta organización de la forma siguiente: "El material ha sido agrupado de una forma orgánica que permite descubrir las relaciones internas entre los distintos manuscritos y resalta la homogeneidad del contenido de la biblioteca qumránica. El primer capítulo contiene las principales Reglas sectarias, los documentos que más informaciones nos proporcionan sobre la estructura, forma de vida y de gobierno, prácticas y pensamiento, de la comunidad o comunidades a las que van dirigidos. El segundo capítulo recoge textos igualmente normativos, los textos halákicos, que nos muestran de manera concreta la aplicación peculiar de la legislación veterotestamentaria vigente dentro del grupo. El tercer capítulo agrupa composiciones muy diversas, pero que comparten todas ellas una misma temática central en las preocupaciones de la comunidad qumránica: la exploración de las realidades de "los últimos tiempos". El capítulo cuarto recoje la producción exegética de la comunidad, aquellas composiciones dedicadas expresamente a mostrarnos cómo el texto bíblico era interpretado, traducido, e incluso transformado. El capítulo quinto agrupa toda una gran serie de creaciones literarias que he designado como "literatura para-bíblica"; se trata de composiciones paralelas al texto bíblico, más o menos cercanas al texto de orígen, o que representan tradiciones autónomas desarrolladas en torno a las figuras bíblicas, o que nos revelan otras creaciones literarias del mismo tipo que las narraciones bíblicas pero que no tuvieron la fortuna de ser recogidas dentro de la Biblia, aunque alguna de ellas, como el Libro de los Jubileos, parecen haber gozado dentro de la comunidad de una

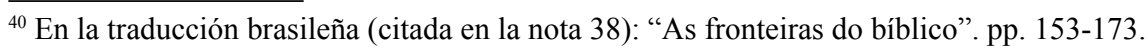


verdadera autoridad bíblica. El capítulo sexto recoge aquellas composiciones poéticas cuyo empleo litúrgico no es seguro, Salmos Apócrifos, Hodayot, poemas sapienciales, etc., mientras que el capítulo séptimo contiene aquellas obras que conservan indicios de haber sido destinadas a una utilización litúrgica o que son restos de rituales. En el capítulo octavo están representadas las composiciones astronómicas, calendarios y horóscopos hallados en las cuevas, obras todas ellas de una importancia religiosa fundamental para la comunidad qumránica. El capítulo noveno contiene un solo documento: el Rollo de Cobre, un texto único en su género y cuyo significado sigue siendo extremadamente problemático." ${ }^{41}$ Es decir, que mi agrupación y caracterización de los textos "no-bíblicos" se entroncaba en la visión canónica corriente, a pesar de que en mi artículo de 1991 yo comenzaba a articular ciertas reservas.

\section{Perspectivas actuales}

Una vez que todos los manuscritos han sido publicados el empleo de las categorías de "textos bíblicos" y "textos no bíblicos" ha perdido toda su utilidad, puesto que ha quedado claro que a nivel de la biblioteca de Qumrán no podemos aún hablar de "Biblia". ${ }^{42}$ Es cierto que entre los manuscritos encontrados hay numerosas copias de determinados libros que forman parte de la Biblia hebrea (un 20 por ciento del total de los manuscritos). Pero estos manuscritos muestran claramente que el proceso de canonización de los escritos que eventualmente formarán la Biblia judía no se había concluído aún. Si entendemos canon en sentido moderno (que implica un número fijo de libros y una forma textual igualmente fija de cada uno de esos libros cuya colección forma "la Biblia,") es obvio que en el contexto de Qumrán no se puede hablar de canon en este sentido. Canon, según la definición de Ulrich, presupone una decisión por la que se llega a un acuerdo comunitario y oficial de que ciertos libros son obligatorios para una comunidad concreta. ${ }^{43}$ En consecuencia, canon denota

${ }^{41}$ Textos de Qumran, 12-13, en la traducción brasileña, pp. 10-11.

${ }^{42}$ Ver GARCÍA MARTÍNEZ, F. "Rethinking the Bible: Sixty Years of Dead Sea Scrolls Research and Beyond”. En POPOVIĆ, M. (ed.), Authoritative Scriptures in Ancient Judaism. JSJSup. 141; Leiden: Brill, 2010, pp.16-36.

${ }^{43}$ ULRICH, E. "The Canonical Process, Textual Criticism, and Latter Stages in the Composition of the Bible". En FISHBANE, M. y TOV, E. (eds.), "Sha 'arei Talmon" Studies in the Bible, Qumran, and the Ancient Near East Presented to Shemaryahu Talmon. Winona Lake, Eisenbrauns, 1992, pp. 267-291, reimpreso en The Dead Sea Scrolls and the Origins of the Bible. SDSSRL; Grand Rapids: Eerdmans, 1999, pp. 51-78. 
una lista cerrada que especifica qué libros son incluídos o excluídos de la Biblia de esa comunidad. En la colección de manuscritos de Qumrán no encontramos ninguna traza de estos presupuestos fundamentales. No sólo no encontramos ninguna lista de libros incluídos o excluídos, sino que tanto las composiciones que posteriormente formarán la Biblia judía, como las obras que no llegarán a formar parte de ella muestran una extraordinaria pluralidad textual. ${ }^{44}$ Esto aparece con toda claridad, cuando se comparan los textos "bíblicos" de la colección de Qumrán con los textos "bíblicos" de las otras colecciones de manuscritos hallados en otros lugares del desierto de Judá (como en las Cuevas de Murabba at o del Nahal Hever) y que son claramente posteriores. En estas otras colecciones el texto bíblico se halla ya cristalizado, y es prácticamente idéntico al texto de nuestras biblias hebreas. Mientras que en Qumrán, a diferencia de las otras colecciones, encontramos muchos manuscritos "bíblicos" y en muchas formas diferentes (en formas textuales claramente distintas, en distintas ediciones o reescritos para formar nuevas composiciones) y todas ellas usadas indiscriminadamente. Si los dos grandes manuscritos de Isaías de la cueva 1 eran del tipo textual que llamamos "proto-masorético," las otras cuevas nos han ofrecido muchos textos del tipo "proto-septuaginta" o del tipo "proto-samaritano" y aún muchos más manuscritos que no pueden alinearse con ninguno de los tres tipos clásicos del texto bíblico, pues mezclan características y lecturas de estos tres tipos textuales, que atestiguan diversas ediciones del texto bíblico, o simplemente presentan una pluralidad textual inesperada. Esta variedad de textos bíblicos, evidente una vez que el conjunto ha sido publicado, nos muestra que antes del establecimiento de un texto único reconocido como autoritativo existía una gran variedad de textos que convivían con la misma autoridad en un mismo espacio geográfico y humano. Es decir que entre los años 70 y 130 de nuestra era, dentro de Judaísmo palestino se ha completado el proceso

\footnotetext{
${ }^{44}$ Sobre este problema, ver las posiciones contrapuestas de VAN DER WOUDE, A.S. "Pluriformity and Uniformity: Reflections on the Transmission of the Text of the Old Testament". En BREMMER, J.N. y GARCÍA MARTÍNEZ, F. (eds.), Sacred History and Sacred texts in Early Judaism: A Symposium in Honour of A.S. van der Woude. Contributions to Biblical Exegesis and Theology 5; Kampen: Kok-Pharos, 1992, pp. 151-169, y de LANGE, A. "“Nobody Dared to Add to Them, to Take from Them, or to Make Changes' (Josephus, Ag. Ap. 1.42). The Textual Stadardization of Jewish Scriptures in Light of the Dead Sea Scrolls". En HILHORST, A., PUECH, E. y TIGCHELAAR, E. (eds.), Flores Florentino: Dead Sea Scrolls and Other Early Jewish Studies in Honour of Florentino Garcia Martínez. JSJS 122, Leiden: Brill, 2007, pp. 105-126, así como mi crítica de ambas posiciones en "Rethinking the Bible" (citado en la nota 42), pp. 24-28.
} 
de canonización que lleva a la formación de la Biblia judía, cosa que no ha sucedido aún al interior de la colección qumránica, que es claramente anterior a los que Shemaryahu Talmon llama "the Great Divide". ${ }^{45}$ Lo que nos obliga a transformar nuestras categorías respecto a la normatividad de los textos sagrados y nuestra comprensión del proceso de formación de textos canónicos; y nos obliga a relativizar tanto el concepto de texto "bíblico" como el de texto "para-bíblico" al interior de la colección qumránica, una colección de textos religiosos en la que el concepto de "Biblia" es anacrónico. ${ }^{46}$

Un ejemplo que prueba esto de manera totalmente clara es el manuscrito de la cueva 4 conocido como 4QTestimonia (4Q175). ${ }^{47}$ Esta hoja contiene una colección de cuatro citas, sin comentario o explicación alguna, aunque cada una claramente marcada por un espacio en blanco y por signos marginales. Lógicamente podemos concluir que estas cuatro citas, que se hallan las cuatro al mismo nivel y son precedidas de las mismas fórmulas de introducción, fueron recogidas para proporcionar pruebas de las ideas de quien las recogió (en este caso el mismo escriba que copió la Regla de la Comunidad y de $4 Q_{\text {Samuel }}^{\mathrm{c}}$ y que emplea el mismo sistema de cuatro puntos para reemplazar el tetragrammaton ${ }^{48}$ ) y nos muestran por tanto la autoridad que se reconoce a los escritos que se aducen como pruebas de estas ideas. Los cuatro textos citados como autoridad son (en este orden): - una cita de Exodo 20:1b según la tradición samaritana, que une y harmoniza Deuteronomio 5:2-29 y Deuteronomio 18:18-19 del texto masorético y anuncia la venida de un profeta como Moisés; - la segunda cita proviene de Números 24:15-17 en una forma textual semejante a la del texto masorético, pero con varias diferencias (no sólo ortográficas, sino substanciales) y que interpreta el oráculo de Balaam sobre el "cetro y la estrella"como refiriéndose a una futura figura mesiánica;

${ }^{45}$ TALMON, Sh. " The Crystallization of the 'Canon of Hebrew Scriptures' in the Light of Biblical Scrolls from Qumran". En HERBERT, E.D. y TOV, E. (eds.), The Bible as Book. The Hebrew Bible and the Judaean Desert Discoveries. London-New Castle: The British LibraryOak Knoll, 2000, pp. 5-20 (en la página 14).

${ }^{46}$ Ver GARCÍA MARTÍNEZ, F. "Parabiblical Literature from Qumran and the Canonical Process". Revue de Qumrân 25/100 (2012), pp. 525-556.

${ }^{47}$ Editado por ALLEGRO J.M. En DJD 5, 57-60, pl XXI (citado en la nota 12). El manuscrito ha sido estudiado intensamente. Para un bibliografía selecta, ver STEUDEL, A. "Testimonia". En SCHIFFMAN, L.H. y VANDERKAM, J. (eds.), Encyclopedia of the Dead Sea Scrolls. New York: Oxford, 2000, pp. 936-938.

${ }^{48}$ Sobre este escriba, ver TIGCHELAAR, E. "In Search of the Scribe of 1QS". En PAUL, Sh.M. y otros (eds.), Emanuel: Studies in Hebrew Bible, Septuagint, and Dead Sea Scrolls in Honor of Emanuel Tov. SVT 94; Leiden: Brill, 2003, pp. 439-452. 
- la tercera cita proviene de Deuteronomio 33:8-11; también presenta algunas variantes con relación al texto masorético y aplica la bendición de Leví al mesías sacerdotal esperado; - la última cita proviene de una composición que era totalmente desconocida hasta que fue descubierta en dos manuscritos qumránicos (4Q378-379) que han sido publicados con el título de 4Qapócrifo de Josué. ${ }^{49}$ Se trata de una composición que reescribe el libro de Josué, mezclando la trama narrativa con plegarias y discursos, la mayoría pronunciados por Josué, como la maldición de Jericó, citada de acuerdo con Josué 6:26. Podemos lógicamente conluir que estas cuatro citas, puestas todas en el mismo nivel, con las misma fórmulas de introducción, eran consideradas como pruebas, tomadas de libros autoritativos para apoyar las ideas del escriba que las coleccionó, y que por tanto pueden enseñarnos algo sobre los escritos considerados como autoritativos dentro de la colección de Qumrán. Estas fuentes autoritativas son: una versión aumentada y harmonizada del libro del Exodo atestiguada en vários manuscritos qumránicos, que terminará por ser parte de la "Biblia" Samaritana y que es considerada por Emanuel Tov como muy cercana a las "composiciones que reescriben la Biblia," 50 es decir, un texto para-bíblico; dos versions ligeramente modificadas de Números y de Deuteronomio, dos libros que terminarán por ser "Biblia" para Judíos y Cristianos; y una composición completamente desconocida, que es clasificada generalmente como "reescritura bíblica," puesto que es secundaria y claramente inspirada en el libro de Josué al que cita pero que aquí es considerada tan autoritativa como los otros tres escritos. En Qumrán, pues, no podemos aún hablar de "canon" y por consiguiente el concepto de "Biblia" es totalmente anacrónico. ${ }^{51}$

La misma pluralidad textual que encontramos en los textos "bíblicos," la encontramos en otras composiciones "no bíblicas" y en los escritos producidos por quienes reunieron los manuscritos como la Regla de la Comunidad, lo

${ }^{49}$ Editados por NEWSOM, C.A. En DJD 22, 237-288, pl. XVII-XXV (ver nota 34). Yo me he ocupado de estos manuscritos en GARCÍA MARTÍNEZ, F. "Light on the Joshua Books form the Dead Sea Scrolls". En AUSLOOS, H., LEMMELIJN, B. y TREBOLLE BARRERA, J. (eds.), After Qumran. Old and Modern Editions of the Biblical Texts - The Historical Books. BETL 246; Leuven: Peeters, 2012, pp. 145-159.

${ }^{50}$ TOV, E. "Rewritten Bible Composition and Biblical Manuscripts, with Special Attention to the Samaritan Pentateuch". Dead Sea Discoveries 5 (1998), pp. 334-354.

${ }^{51}$ Ver GARCÍA MARTÍNEZ, F. "Revelación, Autoridad y Canon en Qumrán”. En MILÁN, F. (ed.), Revelación, Escritura, Interpretación. Estudios en honor del Prof. D. Gonzalo Aranda Pérez. Biblioteca de Teología 35; Pamplona: Eunsa, 2014, pp. 87-108. 
que relativiza la utilidad de la aplicación de etiquetas de género literario a las composiciones contenidas en los manuscritos. ${ }^{52}$ Esta utilización considera un género literario como algo fijo, mientras que en el Judaísmo antiguo lo que caracteriza a las obras literarias es la enorme fluidez y pluralidad textual que encontramos en distintas copias de una misma composición, que tienen a veces diferentes formas e incluso diferentes títulos.

Un buen ejemplo nos lo proporcionan las distintas versiones y los distintos títulos de la composición designada Regla de la Comunidad. El empleo de este género literario "Regla" como etiqueta para designar tanto la Regla de la Comunidad como la Regla de la Congregación está perfectamente justificado, puesto que la palabra "Regla" (סרך) es utilizada en los títulos de ambas composiciones en los manuscritos de la Cueva 1 (סרך היחד ומן, escrito en la parte exterior del manuscrito que contenía ambas composiciones; y osta es la regla para los hombres de la Comunidad" al comienzo de la columna quinta, que corresponde al comienzo de la parte más antigua de la composición antes de los añadidos de las columnas 1-4 de 1Q28; e igualmente en la Regla de la Congregación 1Q28a וזה הסרך לכול עדת ישראל "esta es la regla para toda la Congregación de Israel"). Sin embargo una comparación con los títulos de la misma composición conservados en la cueva 4 nos obliga a relativizar su valor taxonómico. La copia paleográficamente más antigua de las halladas en la cueva 4, 4Q255, ha conservado el título en el incipit, con una adición determinante: libro (ספר): "Libro de la Regla de la Comunidad" (סרך היחד ספר). Uno de los ejemplares paleográficamente más tardío, y cuyo texto comienza con lo que en la copia de la cueva 1 es la columna 5 , y que en realidad representa la forma más antigua de la composición, 4Q258, lleva por título: "Midras para el Instructor sobre los hombres de la ley" (התורה מדרש למשכיל על אנשי), es decir que no posee la etiqueta genérica de serek sino la de midrash, y no se dirige a "los hombres de la Comunidad" sino a "los hombres de la ley." Este ejemplo nos prueba que aunque el empleo de la etiqueta genérica de "Regla" para estas composiciones es perfectamente legítimo y no se halla influído por el empleo posterior cristiano de ese género literario (como en la "Regla de San Pacomio" o la "Regla de San Benito"),

${ }^{52}$ Ver HEMPEL, Ch. "Pluralism and Authoritativeness-The Case of the S Tradition". En Authoritative Scriptures in Ancient Judaism (citado en nota 42), 193-208; eadem, "The Social Matrix That Shaped the Hebrew Bible and Gave Us the Dead Sea Scrolls". En KHAN, G. y LIPTON, D. (eds.), Studies on the Text and Versions of the Hebrew Bible in Honour of Robert Gordon. Leiden: Brill, 2012, pp. 211-237. 
esa clasificación genérica no tenía en la antigua literatura judía la rigidez que tienen ahora, puesto que una "regla" puede ser igualmente definida como "libro" o como "midrash."

Aún más claro me parece el ejemplo siguiente, puesto que esta fluidez se encuentra en el título mismo del manuscrito. Se trata de 4Q249, que contiene restos de una composición en escritura críptica, pero que lleva al dorso el título en caracteres hebreos..$^{53} \mathrm{El}$ título, tal y como puede leerse, es: "Midras del libro de Moisés" (מדרש ספר מושה) una fórmula semejante a la que encontramos en el segundo libro de Crónicas 24:27 מדרש ספר המלכים "Midras del Libro de los Reyes". Pero un nueva fotografía en color hecha recientemente revela dos cosas importantes: - que la palabra "libro" (ספר) ha sido cancelada con tres puntos de cancelación (que es el procedimiento corriente en los manuscritos), - y que la palabra "Midrash" ha sido escrita por una mano distinta a la de ספר מושה , lo que obliga a concluir que el título original de la composición era el de "Libro de Moisés," (un título que aparece también en la "Carta haláquica" 4Q397donde es generalmente considerado como una designación del Pentateuco), y que este título ha sido cambiado posteriormente en "Midrash de Moisés" (מדרש מושה), lo que nos indica una valoración distinta del contenido del mansucrito, puesto que un lector antiguo ha cambiado el título de "libro" por el de "midrash," es decir que ha interpretado el contenido del manuscrito no como un "libro de Moisés" sino como una "interpretación de Moisés." ${ }^{54}$

No es pues extraño que los editores más conscientes de la fluidez de los géneros literarios en la antiguedad judía no hayan dudado en cambiar la designación genérica de los manuscritos que les han sido encomendados. George Brooke es uno de los investigadores que más profundamente ha reflexionado sobre los problema taxonómicos al editar 4Q252, ${ }^{55}$ y aún más explícitamente al discutir la futura reedición de 4Q174 4QFlorilegium. ${ }^{56} \mathrm{La}$ parte final de 4Q252 había sido objeto de una edición preliminar por Allegro con el título de "Bendiciones Patriarcales," puesto que las columnas 4 y 5

\footnotetext{
${ }^{53}$ Citado en la nota 5.

${ }^{54}$ Ver BEN-DOV, J. y STÖKL BEN EZRA, D. “4Q249 Midrash Moshe: A New Reading and Some Implications". Dead Sea Discoveries 21 (2014), pp. 131-149.

${ }^{55}$ En DJD 22 (citado en la nota 34), 185-207, pls. XII-XIII.

${ }^{56}$ BROOKE, G.J. "From Florilegium or Midrash to Commentary: The Problem of Re-naming an Adopted Manuscript". En BROOKE, G.J. y HØGENHAVEN, J. (eds.), The Mermaid and the Partridge: Essays from the Copenhagen Conference on Revisiting Texts from Cave 4. STDJ96; Leiden: Brill, 2011, pp. 129-150.
} 
contienen las bendiciones de Jacob a sus hijos, y en 4:3 después de un vacat que indica el comienzo de una nueva sección leemos efectivamente "Bendiciones de Jacob" (ברכות יעקוב). ${ }^{57}$ Pero Milik, que es quien debía editar el manuscrito completo lo designó como pesher y le dió el nombre de 4QGenesis Pesher $\mathrm{A},{ }^{58}$ puesto que la columna quinta es efectivamente un pesher y la palabra misma aparece en 4:5. Milik pues aplicaba a toda la composición la etiqueta genérica que correspondía a una de las partes. Eisenman y Wise publicaron este texto como "A Genesis Florilegium", que corresponde mejor al contenido de la obra entera que selecciona y comenta distintas partes del Génesis, aunque este título es claramente dependiente de una designación genérica de la literatura cristiana posterior. ${ }^{59}$ Brooke, después de considerar la posibilidad de etiquetarlo como midrash, le ha dado el título más general de "Comentario" y ahora su designación oficial es 4QCommentary on Genesis A. El mismo Brooke, que prepara la reedición de 4QFlorilegium del volumen DJD 5, ha decidido cambiar este título en la nueva edición por el de 4QComentario escatológico A. ${ }^{60}$

En el Prólogo a los Textos de Qumrán yo explicaba los títulos dados a los manuscritos de este modo: "La misma finalidad práctica y de servicio al lector me ha decidido a dotar a cada obra de un título que permita una identificación más fácil de retenir que la del número de serie de publicación oficial .... Para evitar confusiones he mantenido los títulos asignados por los editores aún cuando son claramente inadecuados. En los demás casos he procurado que el título atribuído describa de alguna forma el contenido del manuscrito o su género literario". ${ }^{61}$ Por supuesto, poner un nombre a una obra literaria o cambiárselo no es una actividad anodina e inocua sino que puede condicionar profundamente la manera de compreder esa obra literaria. Este es el motivo por el que en la edición bilingüe Study Edition de 1997-1998 y en la re-edición en paperback de 2000, yo abandoné completamente el intento de agrupación de los manuscritos de acuerdo con su contenido o según su supuesto género literario, limitándome a editarlos siguiendo el orden de las distintas cuevas en los que

${ }^{57}$ ALLEGRO, J.M. "Further Messianic References in Qumran Literature”. Journal of Biblical Literature 75 (1956), pp. 174-187.

${ }^{58}$ MILIK, J.T. "Milkî-ședeq et Milkî-reša dans les anciens écrits juifs et chrétiens”. Journal of Jewish Studies 23 (1972), p. 138.

${ }^{59}$ EISENMAN, R.H. y WISE, M.O. The Dead Sea Scrolls Uncovered. Shaftesbury: Element Books, 1992, pp. 86-87.

${ }^{60}$ BROOKE, G.J. "From Florilegium or Midrash to Commentary." (citado en nota 56), p. 150.

${ }^{61}$ Textos de Qumran, p. 11, en la traducción brasileña, p. 9. 
fueron hallados y de acuerdo con el número de serie de la publicación oficial. ${ }^{62}$ En esta publicación el número de serie es seguido por la sigla del manuscrito de acuerdo con el "Inventory of the Qumran Texts" de Emanuel Tov, ${ }^{63}$ y del título que los editores han dado a las composiciones en la edición oficial en DJD. Pero el elemento central es la identificación del manuscrito por su número de serie, para evitar el condicionar la interpretación que se puede dar a su contenido.

Moshe Bernstein, que prepara la reedición de 4QOrdenanzas ha escrito: "Even though one might argue that scholars should know better than to be misled by the titles created in modernity, we all know that names can be very influential upon the ways in which later scholarship will approach a text." ${ }^{64}$ En cuanto editor de los manuscritos de la Cueva 11 en DJD 23, ${ }^{65}$ yo soy muy consciente de que el título dado a un manuscrito, puede dirigir la manera en la que intentamos comprender su contenido. 11Q29 es un fragmento minúsculo e insignificante, pero su título (perfectamente justificado) "11QFragmento relacionado con la Regla de la Comunidad" le da una importancia mucho mayor de la que en realidad tiene. En esa edición oficial de los materiales del lote holandés hemos seguido el principio de respetar los título dados en las ediciones preliminares aunque no estuvieramos de acuerdo con ellos. Así 11Q21 lleva el título de 11QTemplec? Este manuscrito había sido publicado por Elisha Qimron como una tercera copia del Rollo del Templo ${ }^{66}$ y ha conservado este título en la edición oficial, aunque el punto de interrrogación muestra las dudas en cuanto a lo ajustado del título. Cambiamos el título de 11Q14, que había sido publicado por Van der Woude como 11QBerakot (11QBendiciones) ${ }^{67}$ en 11QSefer ha-Milhamah (11QLibro de la Guerra), cuando quedó claro que el manuscrito contenía la misma composición que 4Q285, y que esta obra iba

${ }^{62}$ GARCÍA MARTÍNEZ, F. y TIGCHELAAR, E.J.C. The Dead Sea Scrolls Study Edition. Leiden-Grand Rapids; Brill-Eerdmans, 2000.

${ }^{63}$ DJD 39 (citado en nota 24), pp. 27-114

${ }^{64}$ BERNSTEIN, M.J. “4Q159: Nomenclature, Text, Exegesis, Genre”. en The Mermaid and the Partridge (citado en nota 56), p. 34.

${ }^{65}$ GARCÍA MARTÍNEZ, F., TIGCHELAAR, E.J.C. y VAN DER WOUDE, A.S. Qumran Cave 11.II. DJD 23; Oxford; Clarendon, 1998.

${ }^{66}$ QIMRON, E. The Temple Scroll. A Critical Edition with Extensive Reconstructions. Judean Desert Studies; Beer-Sheva-Jerusalem, Ben Gurion University of the Negev Press-Israel Exploration Society, 1996.

${ }^{67}$ VAN DER WOUDE, A.S. "Ein neuer Segensspruch aus Qumran (11QBer)". En S. Wagner (ed.), Bibel und Qumran. Beiträge zur Erforschung der Beziehungen zwischen Bibel- und Qumranwissenschaft. Hans Bardtke zum 22.9.1966. Berlin: Evangelische Haupt-Bibelgesellschaft, 1968, pp. 253-258. 
a ser publicada por Alexander y Vermes con ese título. ${ }^{68}$ Pero $11 \mathrm{Q} 10$ ha sido publicado como 11QTargum de Job, aunque sea completamente diferente de los targumes rabínicos, y 11Q11 sigue siendo designado como 11QSalmos Apócrifos, aunque una designación como "Salmos de exorcismos" le convendría mejor y no sería peyorativa.

Tan problemática como la etiquetación de parte de los manuscritos como "manuscritos bíblicos" y de otros manuscritos como "no bíblicos" resulta la etiquetación de ciertos manuscritos como "sectarios" y otros como "no sectarios." La questión de precisar los criterios que permiten atribuir una de esas etiquetas a determinadas composiciones es algo que ha dividido a los investigadores desde los comienzos, desde el famoso artículo de Carol Newsom, ${ }^{69}$ y los subsequentes estudios de Dimant, Lange y Hempel, ${ }^{70}$ hasta el más reciente intento de Jutta Jokiranta. ${ }^{71}$ Independientemente del problema de emplear la categoría sociológica de "secta" para designar una categoría literaria: ¿Cómo determinar qué composiciones son producto del grupo qumránico y reflejan por tanto las idea propias y eventualmente peculiares de este grupo, y qué composiciones han sido producidas fuera del grupo y pueden considerarse por tanto como representativas de otras corrientes dentro del Judaísmo o como producto del Judaísmo de la época en general?

Hace ya algunos años yo proponía como posible solución para resolver estos dilemas aplicar a estos textos la misma perspectiva que ha permitido resolver la dicotomía "bíblico - no bíblico," es decir ver los textos en su contexto propio y no desde perspectivas posteriores, observar las estrategias empleadas para afirmar la autoridad de esos escritos, y reconocer que en gran manera esta distinción entre "sectario y no-sectario" es artificial y producto de nuestra incapacidad a hacer abstracción de nuestras propias categorías a la hora de comprender la realidad histórica que la colección de manuscritos nos

${ }^{68}$ En ALEXANDER, P. y otros, Qumran Cave 4.XXVI. DJD 36; Oxford: Clarendon, 2000,pp. 228-246, pls. XII-XIII.

${ }^{69}$ NEWSOM, C.A. “'Sectually Explicit' Literature from Qumran”. En PROPP, W.H., HALPERN, B. y FREEDMAN, D.N. (eds.), The Hebrew Bible and its Interpreters. Winona Lake: Eisenbrauns, 1990, pp. 167-187.

${ }^{70}$ DIMANT, D. "The Qumran Manuscripts: Contents and Significance". En DIMANT, D. y SCHIFFMAN, L.H. (eds.), Time to Prepare the Way in the Wilderness. STDJ 16; Leiden: Brill, 1995, pp. 25-58; LANGE, A. Weisheit un Prädestination. STDJ 18; Leiden: Brill, 1995, pp. 6-20; HEMPEL, Ch. "Kriterien zur Bestimmung essenischer Verfasserschaft von Qumran Texten". En FREY, J. y STEGEMANN, H. (eds.), Qumrankontrovers. Paderborn: Bonifacius, 2003, pp. 71-85. ${ }^{71}$ JOKIRANTA, J. Social Identity and Sectarianism in the Qumran Movement. STDJ 105; Leiden: Brill, 2013. 
presenta. ${ }^{72}$ Ahora que conocemos la totalidad de los manuscritos preservados, queda totalmente claro que toda la colección qumránica de manuscritos está formada por textos religiosos en cuya formación han influído otros textos precedentes considerados como más o menos autoritativos. ${ }^{73}$

\section{Conclusión}

Lo que me permite concluir con una proposición coherente y, en el fondo, bastante simple, para resolver el problema taxonómico: la de abandonar los esfuerzos de clasificación anacrónicos de los manuscritos de la colección qumránica como textos "bíbiblicos o no- bíblicos" y "sectarios o no-sectarios," y la de considerar el conjunto de la collección como un conglomerado de textos religiosos más o menos autoritativos para el grupo que los recogió, los conservó y, en determinados casos, los compuso. La evidencia conservada, esencialmente circunstancial y fragmentaria, no nos permitirá en cada caso determinar el nivel de autoridad de una composición concreta, pero nos evitará ciertamente los problemas inútiles que resultan de nuestro enfrentarnos a la evidencia conservada desde la óptica de una época completamente distinta de aquella en la que estos escritos fueron creados, copiados y leídos.

En definitiva, lo que esta propuesta implica es el abandonar la perspectiva condicionada por los avatares de transmisión posterior en un medio ambiente judío en el caso de ciertos escritos, o en un ambiente cristiano en el caso de otros, para tomar realmente en serio el hecho de que esta colección de manuscritos nos ofrece una ocasión única de apreciar en un contexto histórico concreto la manera en la que un grupo judío en Palestina en la época del Secundo Templo se enfrentaba con el conjunto de escritos religiosos que gobernaban e inspiraban su vida.

No creo que pueda haber ninguna duda de que el grupo que recogió, conservó y, en ciertos casos, compuso los manuscritos que nos han llegado fragmentariamente, reconocía como supremamente autoritativos los libros de la Torah mosáica en las distintas formas en las que nos han llegado, así como los libros de los Profetas en sus distintas formas, y que estos escritos eran

\footnotetext{
${ }^{72}$ GARCÍA MARTÍNEZ, F. “¿Sectario, no-sectario, o qué? Problemas de una taxonomía correcta de los textos qumránicos". Revue de Qumrân 23/91 (2008), pp. 383-94

${ }^{73}$ GARCÍA MARTÍNEZ, F., "I testi qumranici testimoni di Scriture autorevoli." En. PRATO, G.-L. (ed.), Convegno della AIG 2009. Ricerche Storico Bibliche. Bologna: Dehoniane, 2011, pp. 17-33.
} 
igualmente reconocidos como autoritativos por otras corrientes judías de la época. Tampoco creo que pueda haber grandes dudas de que el grupo qumránico reconocía igualmente como autoritativos (aunque sea difícil el precisar el grado exacto de autoridad atribuído en cada caso) otras composiciones que gozaban de autoridad en ciertos grupos judíos pero no en otros, como Jubileos, el Documento arameo de Leví, o el Rollo del Templo. Y me parece evidente que los escritos de producción propia, como las Reglas,los Himnos, el Documento de Damasco y los escritos en los que interpretaban la propia historia a la luz de los libros de los Profetas, es decir los escritos en los que extendían, desarrollaban y profundizaban su comprensión peculiar de todos esos escritos autoritativos (fueran reconocidos como tales o no por otros grupos Judíos) eran igualmente considerados como autoritativos por ellos (aunque en muchos casos sea imposible de precisar en qué grado).$^{74} \mathrm{El}$ abandono de los intentos de clasificación de estos escritos de acuerdo con nuestras concepciones modernas puede beneficiar nuestra comprensión de la colección en su conjunto y en su contexto histórico real, anterior a la destrucción del Templo y al establecimiento del Judaísmo y del Cristianismo que nosotros conocemos.

\section{Referencias bibliográficas}

ALEXANDER, P. y VERMES, G. Qumran Cave 4. XIX: Serek ha-Yahad and Two Related Texts. DJD 26; Oxford: Clarendon, 1998, 93 pl. X.

ALEXANDER, P. y otros, Qumran Cave 4.XXVI. DJD 36; Oxford: Clarendon, 2000,pp. 228-246, pls. XII-XIII.

ALLEGRO, J.M. "Further Messianic References in Qumran Literature". Journal of Biblical Literature 75 (1956), pp. 174-187.

ALlEGRO, J.M. Qumran Cave 4. I. DJD 5; Oxford: Clarendon, 1969, pp. 77-79, pl. XXVIII.

ARANDA PÉREZ, G., GARCÍA MARTÍNEZ, F. y PÉREZ FERNÁNDEZ, M. Introducción al Estudio de la Biblia, Vol. IX: Literatura Judia Intertestamentaria. Estella: Verbo Divino, 1996.

\footnotetext{
${ }^{74}$ Como creo haber probado en GARCÍA MARTÍNEZ, F. "Beyond the Sectarian Divide: The 'Voice of the Teacher' as Authoritative Conferring Strategy". En METSO, S., NAJMAN, H. y SCHULLER, E. (eds.), The Dead Sea Scrolls: Transmission of Traditions and Production of Texts. STDJ 92; Leiden: Brill, 2010, pp. 227-244.
} 
ATTRIDGE, H. y otros, Qumran Cave 4.VIII: Parabiblical Texts, Part 1. DJD 13; Oxford: Clarendon, 1994.

AVIGAD, N. y YADIN, Y. A Genesis Apocryphon. A Scrolls from the Wilderness of Judaea. Jerusalem: Magnes Press/Heikal ha-Sefer, 1956

BAILLET, M., MILIK,J.T. y DE VAUX, R., Les 'Petites Grottes'de Qumrân. Exploration de la falaise. Les grottes 2Q, 3Q, 5Q, 6Q, 7Q à 10Q. DJD 3; Oxford: Clarendon, 1962.

BAILLET, M. Qumrân grotte 4.III. DJD 7; Oxford: Clarendon, 1982, pp.137168, pl. XLIX-LIII.

BARTHÉLEMY, D. Qumrân Cave 1. DJD 1; Oxford: Clarendon, 1955, pp. 107-118, pls XXIII-XXIV.

BAUMGARTEN, J.M. Qumran Cave 4. XIII: The Damascus Document. DJD 18; Oxford: Clarendon, 1996.

BAUMGARTEN, J. y otros, Qumran Cave 4.XXV: Halakhic Texts._DJD 35; Oxford: Clarendon, 1999.

BEN-DOV, J. y STÖKL BEN EZRA, D. "4Q249 Midrash Moshe: A New Reading and Some Implications". Dead Sea Discoveries 21 (2014), pp. 131-149.

BERNSTEIN, M.J. "4Q159: Nomenclature, Text, Exegesis, Genre”. En BROOKE, G.J. y HØGENHAVEN, J. (eds.), The Mermaid and the Partridge: Essays from the Copenhagen Conference on Revisiting Texts from Cave 4. STDJ96; Leiden: Brill, 2011, p. 34

BROOKE, G.J. y otros, Qumran Cave 4.XVII: Parabiblical Texts, Part 3. DJD 22; Oxford: Clarendon, 1996.

BROOKE, G.J. "From Florilegium or Midrash to Commentary: The Problem of Re-naming an Adopted Manuscript". En BROOKE, G.J. y HØGENHAVEN, J. (eds.), The Mermaid and the Partridge: Essays from the Copenhagen Conference on Revisiting Texts from Cave 4. STDJ96; Leiden: Brill, 2011, pp. 129-150.

BROSHI, M. y otros, Qumran Cave 4.XIV: Parabiblical Texts, Part 2. DJD 19; Oxford: Clarendon, 1995.

BURROWS, M. The Dead Sea Scrolls of St. Mark's Monastery: Volume I. New Haven: The American Scools of Oriental Research, 1950 
BURROWS, M. The Dead Sea Scrolls of St. Mark's Monastery: Volume II. Fascicle 2. New Haven: The American Scools of Oriental Research, 1951.

CHAZON, E. y otros, Qumran Cave 4.XX: Poetical and Liturgical Texts, Part 2. DJD 29; Oxford: Clarendon, 1999.

DAVILA, J.R. Qumran Cave 4. VII: Genesis to Numbers. DJD 12; Oxford: Clarendon, 1995,pp. 63-64, pl. XII.

DIMANT, D. "The Qumran Manuscripts: Contents and Significance". En DIMANT, D. y SCHIFFMAN, L.H. (eds.), Time to Prepare the Way in the Wilderness. STDJ 16; Leiden: Brill, 1995, pp. 25-58;

DIMANT, D. Qumran Cave 4.XXI: Parabiblical Texts, Part 4: PseudoProphetic Texts. DJD 30; Oxford : Clarendon, 2001.

EISENMAN, R.H. y WISE, M.O. The Dead Sea Scrolls Uncovered. Shaftesbury: Element Books, 1992, pp. 86-87.

ELGVIN, T. y otros, Qumran Cave 4.XV: Sapiential Texts, Part 1. DJD 20; Oxford: Clarendon, 1997.

ESHEL, E. y otros, Qumran Cave 4.VI: Poetical and Liturgical Texts. Part I. DJD 11; Oxford: Clarendon 1998.

GARCÍA MARTÍNEZ, F. "Orígenes del movimiento esenio y orígenes qumránicos: Pistas para una solución”. En COLLADO BERTOMEU,V. y VILLAR HUESO, V. (ed.). II Simposio Bíblico Español (Córdoba, 1985). Valencia-Córdoba: Fundación Bíblica Española, 1987, pp. 527-556,

GARCÍA MARTÍNEZ, F. "Qumran Origins and Early History: A Groningen Hypothesis". Folia Orientalia 25 (1988), pp. 113-36,

GARCÍA MARTÍNEZ, F. “Las fronteras de «lo bíblico»”. Scripta Theologica 23 (1991), pp. 759-784.

GARCÍA MARTÍNEZ, F.Textos de Qumrán. Estructuras y Procesos / Serie Religión; Madrid: Editorial Trotta, 1992.

GARCÍA MARTÍNEZ, F. y TREBOLLE BARRERA, J. Los Hombres de Qumrán: Literatura, estructura social y concepciones religiosas. Estructuras y Procesos / Serie Religión; Madrid: Editorial Trotta, 1993.

GARCÍA MARTÍNEZ, F., TIGCHELAAR, E.J.C. y VAN DER WOUDE, A.S. Qumran Cave 11.II. DJD 23; Oxford; Clarendon, 1998. 
GARCÍA MARTÍNEZ, F. y TIGCHELAAR, E.J.C. The Dead Sea Scrolls Study Edition. Leiden-Grand Rapids; Brill-Eerdmans, 2000.

GARCÍA MARTÍNEZ, F. “¿Sectario, no-sectario, o qué? Problemas de una taxonomía correcta de los textos qumránicos". Revue de Qumrân 23/91 (2008), pp. 383-94

GARCÍA MARTÍNEZ, F. "Rethinking the Bible: Sixty Years of Dead Sea Scrolls Research and Beyond". En POPOVIĆ, M. (ed.), Authoritative Scriptures in Ancient Judaism. JSJSup. 141; Leiden: Brill, 2010, pp.16-36.

GARCÍA MARTÍNEZ, F. "Beyond the Sectarian Divide: The "Voice of the Teacher' as Authoritative Conferring Strategy". En METSO, S., NAJMAN, H. y SCHULLER, E. (eds.), The Dead Sea Scrolls: Transmission of Traditions and Production of Texts. STDJ 92; Leiden: Brill, 2010, pp. 227-244.

GARCÍA MARTÍNEZ, F., "I testi qumranici testimoni di Scriture autorevoli." En. PRATO, G.-L. (ed.), Convegno della AIG 2009. Ricerche Storico Bibliche. Bologna: Dehoniane, 2011, pp. 17-33.

GARCÍA MARTÍNEZ, F. "Parabiblical Literature from Qumran and the Canonical Process". Revue de Qumrân 25/100 (2012), pp. 525-556.

GARCÍA MARTÍNEZ, F. "Light on the Joshua Books form the Dead Sea Scrolls". En AUSLOOS, H., LEMMELIJN, B. y TREBOLLE BARRERA, J. (eds.), After Qumran. Old and Modern Editions of the Biblical Texts The Historical Books. BETL 246; Leuven: Peeters, 2012, pp. 145-159.

GARCÍA MARTÍNEZ F. y VERVENNE, M. “Ancient Interpretation of Scripture in Light of the Dead Sea Scrolls." En PIQUER OTERO, A. y TORIJANO MORALES, P. (eds.), Textual Criticism and Dead Sea Scrolls Studies in Honour of Julio Trebolle Barrera: Florilegium Complutensis. JSJS 157: Leiden: Brill, 2012, pp. 83-97.

GARCÍA MARTÍNEZ, F. "Revelación, Autoridad y Canon en Qumrán”. En MILÁN, F. (ed.), Revelación, Escritura, Interpretación. Estudios en honor del Prof. D. Gonzalo Aranda Pérez. Biblioteca de Teología 35; Pamplona: Eunsa, 2014, pp. 87-108.

HEMPEL, Ch. "Pluralism and Authoritativeness-The Case of the S Tradition". En POPOVIĆ, M. (ed.), Authoritative Scriptures in Ancient Judaism. JSJSup. 141; Leiden: Brill, 2010, 193-208. 
HEMPEL, Ch. "Kriterien zur Bestimmung essenischer Verfasserschaft von QumranTexten". En FREY, J. y STEGEMANN, H. (eds.), Qumrankontrovers. Paderborn: Bonifacius, 2003, pp. 71-85.

HEMPEL, Ch. "The Social Matrix That Shaped the Hebrew Bible and Gave Us the Dead Sea Scrolls”. En KHAN, G. y LIPTON, D. (eds.), Studies on the Text and Versions of the Hebrew Bible in Honour of Robert Gordon. Leiden: Brill, 2012, pp. 211-237.

JOKIRANTA, J. Social Identity and Sectarianism in the Qumran Movement. STDJ 105; Leiden: Brill, 2013.

LANGE, A. Weisheit un Prädestination. STDJ 18; Leiden: Brill, 1995, pp. 6-20.

LANGE, A. "“Nobody Dared to Add to Them, to Take from Them, or to Make Changes' (Josephus, Ag. Ap. 1.42). The Textual Stadardization of Jewish Scriptures in Light of the Dead Sea Scrolls". En HILHORST, A., PUECH y TIGCHELAAR, E. (eds.), Flores Florentino: Dead Sea Scrolls and Other Early Jewish Studies in Honour of Florentino Garcia Martínez. JSJS 122, Leiden: Brill, 2007, pp. 105-126.

MACHIELA, D. The Dead Sea Genesis Apocryphon. DTDJ 79; Leiden: Brill, 2009.

MILIK, J.T. Qumrân Cave 1. Discoveries in the Judaean Desert 1; Oxford: Clarendon, 1955, pp. 107-108, pl. XXII.

MILIK, J.T. "Milkî-ṣedeq et Milkî-reša dans les anciens écrits juifs et chrétiens". Journal of Jewish Studies 23 (1972), p. 138.

NAJMAN, H y TIGCHELAAR, E. "A Preparatory Study of Nomenclature and Text Designation in the Dead Sea Scrolls". Revue de Qumrân 103 (2014), pp. 305-325.

NEWSOM, C.A. “'Sectually Explicit' Literature from Qumran”. En PROPP, W.H., HALPERN, B. y FREEDMAN, D.N. (eds.), The Hebrew Bible and its Interpreters. Winona Lake: Eisenbrauns, 1990, pp. 167-187.

PFANN, S. En Qumran Cave 4. XXV: Halakhic Texts. DJD 35; Oxford: Clarendon, 1999, pp. 1-24, pl.I- II.

PHANN, S.J. y KISTER, M. En Qumran Cave 4. XV: Sapiential Texts, Par I. DJD 20; Oxford: Clarendon, 1997, pp. 1-30, pl. I-II. 
PUECH, E. Qumrân grotte 4. XXII: Textes araméens première partie. DJD 31; Oxford: Clarendon, 2001, pp. 1-8, pl. I.

QIMRON, E. The Temple SAcroll. A Critical Edition with Extensive Reconstructions. Judean Desert Studies; Beer-Sheva-Jerusalem, Ben Gurion University of the Negev Press-Israel Exploration Society, 1996.

SCHECHTER, S. En Documents of Jewish Sectaries. Vol. 1: Fragments of a Zadokite Work. Cambridge 1910; New York 1970.

STEGEMANN, H. y SCHULLER, E., 1QHodayot ${ }^{a}$ with incorporation of 1QHodayot ${ }^{b}$ and 4QHodayot ${ }^{a-f}$. DJD 40; Oxford: Clarendon 2009.

STEUDEL, A. "Testimonia". En SCHIFFMAN, L.H. y VANDERKAM, J. (eds.), Encyclopedia of the Dead Sea Scrolls. New York: Oxford, 2000, pp. 936-938.

STRUGNELL, J. y otros, Qumran Cave 4.XXIV: 4QInstruction (Musar leMevin). DJD 34; Oxford: Clarendon, 1999.

SUKENIK, E.L. En The Dead Sea Scrolls of the Hebrew University. Jerusalem: Magnes Press/The Hebrew University, 1955.

TALMON, SH. " The Crystallization of the 'Canon of Hebrew Scriptures' in the Light of Biblical Scrolls from Qumran". En HERBERT, E.D. y TOV, E. (eds.), The Bible as Book. The Hebrew Bible and the Judaean Desert Discoveries. London-New Castle: The British Library-Oak Knoll, 2000, pp. 5-20

TIGCHELAAR, E. "In Search of the Scribe of 1QS". En PAUL, Sh.M. y otros (eds.), Emanuel: Studies in Hebrew Bible, Septuagint, and Dead Sea Scrolls in Honor of Emanuel Tov. SVT 94; Leiden: Brill, 2003, pp. 439-452.

TOV, E. The Texts from the Judaean Desert. Indices and an Introductions to the Discoveries in the Judaean Desert Series. DJD 39; Oxford: Clarendon 2002, pp. 10-14.

TOV, E. "Rewritten Bible Composition and Biblical Manuscripts, with Special Attention to the Samaritan Pentateuch". Dead Sea Discoveries 5 (1998), pp. 334-354.

ULRICH, E. "The Canonical Process, Textual Criticism, and Latter Stages in the Composition of the Bible". En FISHBANE, M. y TOV, E. (eds.), 
"Sha 'arei Talmon" Studies in the Bible, Qumran, and the Ancient Near East Presented to Shemaryahu Talmon. Winona Lake, Eisenbrauns, 1992, pp. 267-291.

ULRICH, E. The Dead Sea Scrolls and the Origins of the Bible. SDSSRL; Grand Rapids: Eerdmans, 1999, pp. 51-78.

ULRICH, E. y FLINT, P.W. En Qumran Cave 1.II: The Isaiah Scrolls. DJD 32; Oxford: Calrendon 2010.

VAN DER WOUDE, A.S. "Pluriformity and Uniformity: Reflections on the Transmission of the Text of the Old Testament". En BREMMER, J.N. y GARCÍA MARTÍNEZ, F. (eds.), Sacred History and Sacred texts in Early Judaism: A Symposium in Honour of A.S. van der Woude. Contributions to Biblical Exegesis and Theology 5; Kampen: Kok-Pharos, 1992, pp. $151-169$.

VAN DER WOUDE, A.S. "Ein neuer Segensspruch aus Qumran (11QBer)". En S. Wagner (ed.), Bibel und Qumran. Beitrâge sur Erforschung der Beziehungen zwischne Bibel- und Qumranwissenschaft. Hans Bardtke zum 22.9.1966. Berlin: Evangelische Haupt-Bibelgesellschaft, 1968, pp. 253-258.

Florentino García Martínez

Doctor en Teología y Ciencias religiosas por la Rijksuniversiteit de

Groningen, Holanda

Catedrático emérito de la universidad de Groningen Emeritus met opdracht de la Katholike Universiteit de Leuven, Bélgica Lovaina / Bélgica e-mail: F.Garcia.Martinez@theo.kuleuven.be

Recebido em: 29/06/15

Aprovado em: 13/08/15 